\title{
Patient-Derived Urothelial Cancer Xenograft Models: A Systematic Review and Future Perspectives
}

\author{
Yuki Kita $^{\mathrm{a}, \mathrm{b}}$, Ryoichi Saito ${ }^{\mathrm{a}}$, Takahiro Inoue ${ }^{\mathrm{a}}$, William Y. Kim ${ }^{\mathrm{b}}$, Osamu Ogawa ${ }^{\mathrm{a}, *}$ \\ and Takashi Kobayashi ${ }^{\mathrm{a}}$ \\ ${ }^{a}$ Department of Urology, Kyoto University Graduate School of Medicine, Kyoto, Japan \\ ${ }^{\mathrm{b}}$ Lineberger Comprehensive Cancer Center, University of North Carolina, Chapel Hill, NC, USA
}

Received 7 February 2020

Accepted 31 March 2020

Pre-press 23 April 2020

Published 11 June 2020

\begin{abstract}
.
BACKGROUND: Lack of appropriate models that recapitulate the diversity, heterogeneity, and tumor microenvironment of urothelial cancer (UC) is a limitation to preclinical models. Patient-derived xenograft (PDX) models are a promising tool to overcome some of these issues, and thus we present an up-to-date and comprehensive overview of UC PDX models to aid in their future use.

OBJECTIVE: To provide an overview on methodology, applications and limitations as well as future perspectives on bladder cancer PDX models.

METHODS: Literature searches using PubMed and Web of Science databases were performed for relevant articles according to the following MeSH terms: "urothelial carcinoma(s)" OR "urothelial cancer" OR "urothelial tumor" OR "bladder cancer(s)" OR "bladder carcinoma(s)" OR "transitional cell carcinoma(s)" AND "xenograft(s)" OR "xenotransplant" at December 6th, 2019. We followed the Preferred Reporting Items for Systematic Reviews and Meta-Analyses guidelines.

RESULTS: Of the 49 studies extracted, 41 studies after the year 2000 were finally analyzed. Published studies show that (1) UC PDX platforms retained the histology and genomic characteristics of the corresponding patient tumors. (2) UC PDX can be applied to ask various questions including to study the mechanisms of disease progression and treatment resistance, to develop novel drugs and biomarkers, as well as to potentially realize personalized drug selection. Recent topics of research using PDX have included the development of humanized mice as well as the use of 3D culture to complement some of the limitations of PDX models.

CONCLUSIONS: UC PDX models serve as tools for understanding cancer biology, drug development and empowering precision medicine. The improvement of experimental systems using humanized mice to recapitulate the immune microenvironment of tumors will optimize UC PDX to study future questions in the field of immunotherapy.
\end{abstract}

Keywords: PDX, precision medicine, 3D culture, humanized mouse, immuno-oncology, urothelial cancer, bladder cancer

\section{INTRODUCTION}

Bladder cancer, with 430,000 new cases and 165,000 deaths per year, stands as the ninth most common cancer worldwide [1]. Muscle-invasive and

${ }^{*}$ Correspondence to: Osamu Ogawa, 54 Shogoinkawahara-cho, Sakyo-ku, Kyoto, 606-8507, Japan. Tel.: +81 757513326; Fax: +81 75 7613441; E-mail: osamuogawa1219@gmail.com. metastatic bladder cancers have a very poor prognosis with 5-year survival rate of $50 \%$ and $15 \%$, respectively [2]. Advanced upper tract cancer, histologically categorized as urothelial carcinoma (UC), is also associated with a poor prognosis. Impressively, treatment outcomes in advanced UC did not improve for close to 30 years until the recent approval of immune checkpoint inhibitors. Despite meaning- 
ful clinical benefits of immune checkpoint inhibitors, the majority of patients still do not respond [3]. One of a number of potential reasons why improvement in treatment outcome for UC has been stagnant is a paucity of appropriate preclinical disease models to use in drug development.

Conventional patient-derived xenograft (PDX) models are established by transferring tumor fragments derived from an individual patient into immunocompromised mice. These models are reported to reflect the underlying biology and heterogeneity of individual tumors more accurately than conventional cell-line-derived xenograft (CDX) models [4-6]. In addition, drug response of PDX models often replicates the clinical response to the same drug in individual donor patients [7, 8]. On the other hand, the low success rate of PDX grafting, the long time required for their establishment, as well as their inability to serve as an evaluation model for immunotherapy have been considered as real deficiencies of PDX models for biological, translational, and pre-clinical UC research. However, the development of new immunodeficient mice has improved the rate of success grafting, and the advent of humanized mice is expected to enable reproduction of the local immunity $[9,10]$. Recent advances in threedimensional (3D) culture methods have also compensated for some of the deficiencies of the PDX model. The PDX and 3D models are therefore complementary and can be integrated to further personalized medicine [11]. In this context, we conducted a systematic review on PDX models of UC to discuss the methodologies, applications and their future as research tools.

\section{METHODS}

This meta-analysis was performed under the Preferred Reporting Items for Systematic Reviews and Meta-Analyses (PRISMA) statement [12]. Literature searches using PubMed and Web of Science databases were performed December 6th, 2019, to identify relevant studies. The following keywords were used for the search: "urothelial carcinoma(s)" OR "urothelial cancer" OR "urothelial tumor" OR "bladder cancer(s)" OR "bladder carcinoma(s)" OR "transitional cell carcinoma(s)" AND "xenograft(s)" OR "xenotransplant". Search restrictions were set for the human species and English language. References cited in the identified literatures were also searched manually to obtain other suitable studies. Each arti- cle was evaluated for inclusion by two of the authors (Y.K. and T.K.) independently and all disagreements were resolved by their consensus. The inclusion criteria for the studies were as follows: (1) establishing patient-derived xenograft (PDX) from pathologically proven human urothelial carcinoma; (2) confirming histological findings on xenograft tumor; (3) reporting some biological or pre-clinical characteristics. The exclusion criteria included: (1) duplicated studies using the same PDX lines; (2) xenograft lines from other cancers or cell-line-derived xenograft (CDX) (3) abstracts, reviews, letters, and comments. The following information was extracted: first author's name, year and journal of publication, country of origin, method for PDX establishment and utilization.

\section{RESULTS}

\section{Search results of literature}

The systematic review process is represented in Fig. 1. A total of 971 articles were identified through PubMed and Web of Science databases, and manual searching on the reference lists of the included studies increased the total references by an additional 4 articles. Of those 975 references, 853 articles were excluded after title and abstract screening and 73 were further excluded after full text analysis. The resulting 49 articles were therefore analyzed for this systematic review.

These 49 reports were divided into two groups: one included 29 papers focused on methods to establish PDX models and the other included 37 papers (partly overlapping with the former) focused on the use of PDX models. Because the use of severe combined immunodeficiency (SCID) mice and its derivatives for PDX generation did not become widespread until the year 2000, we decided to limit our review of the literature to after the year 2000 , resulting in a detailed analysis of 41 reports.

\section{The conventional method of PDX establishment}

A variety of methods to establish UC PDX establishment methods have been reported including the origin of specimen (i.e. TURBT sample or metastatic biopsy), the method of implantation, the host mice and the use of Matrigel, etc. (Table 1). Unfortunately, it is difficult to assess the superiority or inferiority of the differing methods because of the small sample sizes per study as well as a lack of reports that directly 


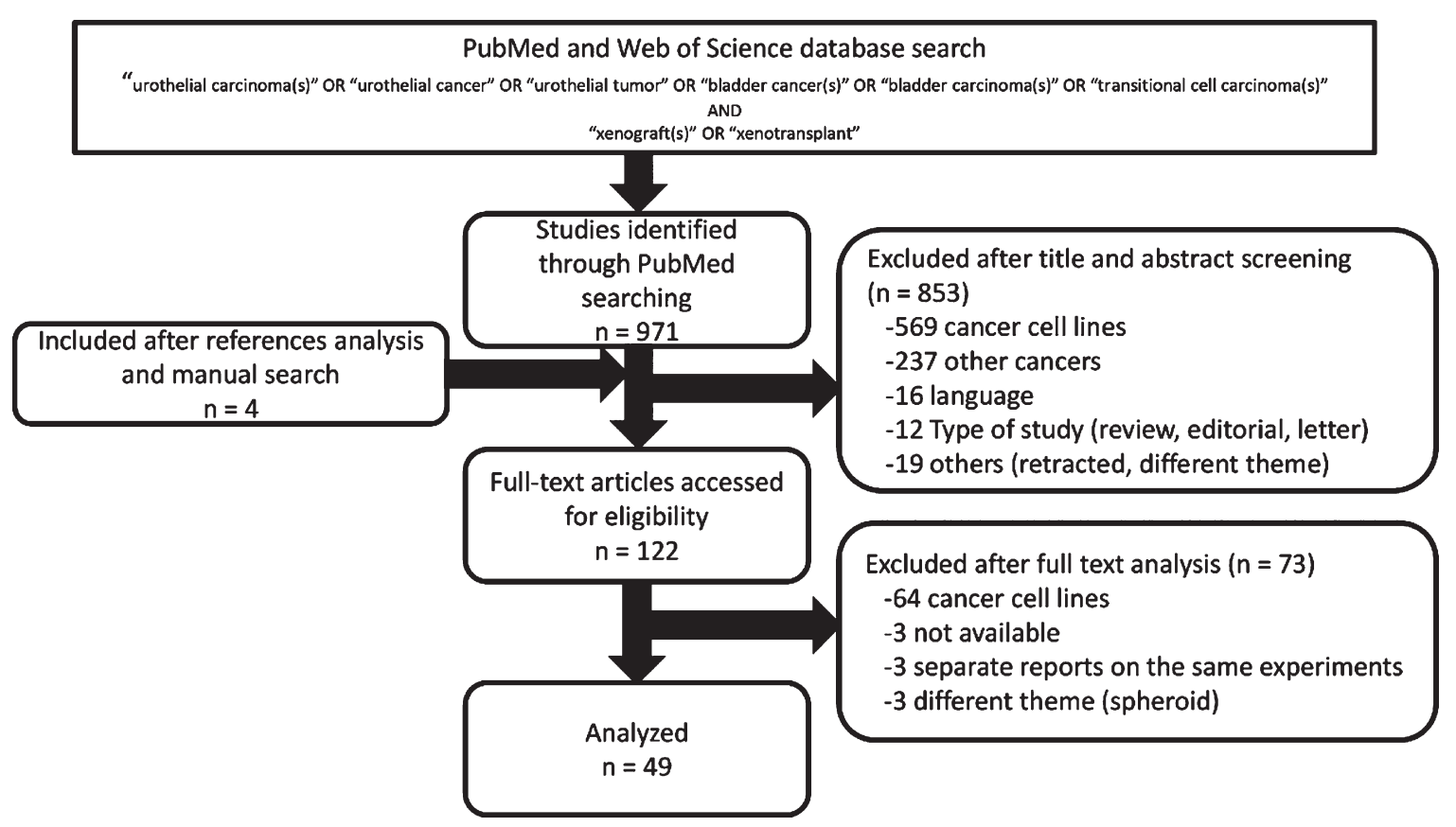

Fig. 1. Flow diagram of the systematic review process.

compare the different methods. That said, the overall engraftment rate, which represents the number of successful tumor growth per patient sample, in reported series with 10 or more clinical specimens ranged from 15 to $60 \%$. In general, immediately after specimen collection and before transplantation, the tumors were maintained in medium containing antibiotics or sterile salt solution. The samples were then cut into 1-3 mm cubes to implant subcutaneously through small dorsal incisions in immunocompromised mice. Implanted tumor fragments often engrafted within about 1 to 3 months, and engraftment failure was judged after 5-6 months of follow-up [13-15]. These grown fragments can be passaged by dividing the excised tumor into smaller pieces and re-implanting them into additional host mice. Efficient cryopreservation protocols for PDX, established in prostate, ovarian and pancreas cancer [16-18], but have not yet been reported for UC. It is necessary to examine whether these methods can be applied to UC PDX.

Immunodeficient mice commonly reported in the literature for UC PDX include nude mice, severe combined immunodeficiency (SCID) mice, nonobese diabetic scid (NOD/SCID) mice and NOD.Cg-Prkdc ${ }^{\text {scid }}$ II2rgt ${ }^{\mathrm{m} 1 \text { Sug }}$ (NOG) mice as well as NOD.Cg-Prdkc ${ }^{\text {scid }} / 12$ rgt $^{\text {m1Wjl }}$ (NSG) mice. SCID mice are deficient in mature $\mathrm{T} / \mathrm{B}$ cells. The NOD/SCID strain not only lack mature T and B cells but also are deficient in macrophages and dendritic cells. NOG and NSG strains carry the NOD/SCID background combined with the IL2 receptor common gamma-chain knockout background and thus also lack NK cells [19].

While never formally directly compared, relative to nude mice, the other strains (SCID and NOD/SCID as well as NOG and NSG) of mice appear to have higher engraftment rates (nude: 15\% (10/65 tumors) [20], SCID; 50\% (15/30) [13], NOD/SCID; 58\% $(51 / 87)[15,21,22]$, NOG and NSG; 40\% (27/68) $[14,23]$. Although the number of samples was small and no direct comparison was made, the use of Matrigel ${ }^{\circledR}$ did not appear to positively affect the engraftment rates (use; $32 \%$ (8/25 tumors), no use; $53 \%$ (85/160) in SCID, NOD/SCID and NSG mice) nor was there a difference in the engraftment rate between placing a tumor fragment under the skin using an incision versus injecting the shredded graft with the needle. It is notable however, that tumors did not establish in any of the three cases where the source of xenografted material was a needle aspiration biopsy sample [22]. Interestingly, transplantation under the renal capsule showed a higher establishment rate than other methods [21]. Muscleinvasive tumors demonstrated higher success rate of establishment than non-muscle-invasive tumors (invasive; $63 \%$ (43/74 tumors), non-invasive; $42 \%$ 


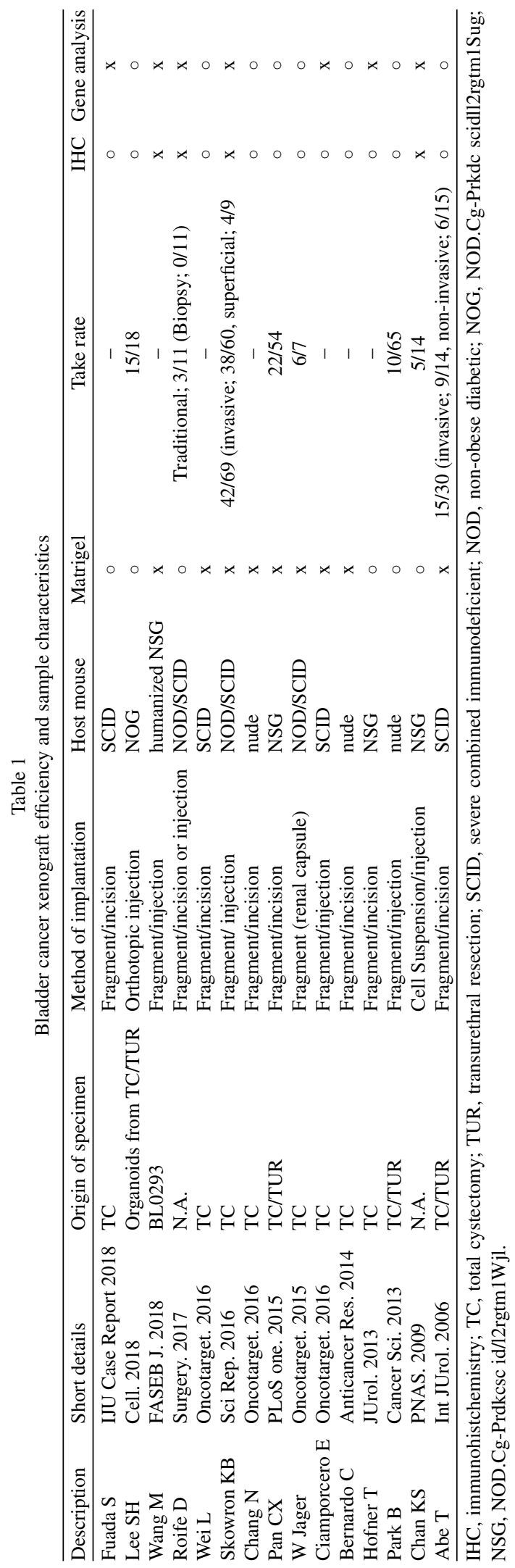

$(10 / 24)[13,15])$. Skowron et al. reported that success of PDX engraftment predicted poor survival in locally advanced bladder cancer [15]. On the other hand, it has been reported that the TP53 mutation status, one of the most frequently mutated genes in bladder cancer, did not correlate with differences in the tumor take rate [13].

It should be noted with caution that subcutaneous tumors differ from orthotopic tumors in terms of intrinsic gene expression profiles and extrinsic pharmacokinetics, as reported in other cancer types [24, 25]. In this regard, however, it is a tough challenge to establish orthotopic PDX from a bladder tumor. Recently, two reports showed that bladder instillation of dissociated cells or cultured cells from once subcutaneously established PDX tumors enabled to create orthotopic PDX models [26, 27]. These models recapitulated invasion from the bladder lumen and metastasis to the lung as well as the architectural and molecular expression characteristics of patient primary tumors. However, further assessment may be needed to determine whether the cell dissociation process necessary for bladder instillation can retain the intratumoral diversity that is an advantage of PDX.

\section{Histologic and Genomic Characterization of PDX models}

Based on hematoxylin and eosin (H\&E) staining with or without immunohistochemical staining, most papers claim that PDX maintain their original tumor morphology. This appears to be true in the case of bladder cancer as well [28]. However, it is imperative that bladder cancer histology be confirmed in UC PDX since Abe et al. reported a number of cases in which engrafted tumors were morphologically different from the original tumors. While the pathologic diagnosis from the patient's tumor was UC, the corresponding PDX turned out to be Epstein-Barr virus (EBV)-associated lymphoproliferative disease [13]. In this case, the authors retrospectively observed EBV-encoded small RNA positive infiltrating lymphocytes in the original tumor, suggesting that the infected lymphocytes were transplanted alongside with the UC cells were able to proliferate neoplasticly in the immunodeficient mice. These PDX lymphocytic tumors have also been reported in PDX models of other cancer types such as breast cancer and pancreatic cancer [29]. Attention should always be paid to the cell morphology of PDX, and if morphological changes are observed, it should be 
confirmed by immunostaining of lymphocytic markers.

In addition to histology, groups have also examined the genomic fidelity of UC PDX to their initial tumor. Several studies analyzing various genomic parameters showed that genetic identity is preserved between UC PDXs and their original tumor samples, based on karyotyping [28, 30-32], flow cytometric quantification of cellular DNA content [31,33], short tandem repeat (STR) polymorphism assay [20], targeted mutational analysis [13, 20, 21], array-based comparative genomic hybridization (aCGH) [20, 21, $34]$, and next-generation sequencing [11, 14, 35]. These studies revealed that the genetic profiles of UC PDXs and their original tumors while not perfectly matched, share very high homology. Jager et al. performed aCGH on $6 \mathrm{UC}$ samples and revealed striking concordance in the copy number profiles between patient tumors and their matched PDX [21]. Interestingly, copy number changes in the PDX models were more prominent, presumably due to reduction in the contribution of human stroma cells. Pan et al. compared whole exome sequencing data of two UC PDXs and original patient tumors to show that they shared $92-97 \%$ of genetic aberrations, including multiple druggable targets [14]. Wei et al. also showed that in two cases, the majority $(91 \%$ and $82 \%$ ) of all mutations were shared by primary and UC PDX samples [35]. Although both comparisons are limited to a small number of cases, these reports prove that UC PDX retains the genetic identity of the primary tumor as reported in other cancer types [7]. In recent years, molecular subtype classification of bladder cancer has attracted attention, however at this time there are no definitive studies addressing whether RNA molecular subtype is conserved in UC PDX tumors.

\section{PDX establishment of rare histologic variants}

For rarer bladder cancer histologic variants that lack other research models (i.e. cell lines or genetically engineered murine (GEM) models), UC PDX models can be very useful tool. Some reports on the establishment of PDX from clinical specimens with rare genotypes or phenotypes have been published. For example, Hofner et al. established neuroendocrine bladder cancer (NEBC) xenograft models from two patient's tumors and identified MET as a potential new treatment target for NEBC [36]. Furthermore, Funada et al. succeeded in establishing a PDX model of IGF-II producing giant cell urothelial carcinoma an aggressive and rare variant of highgrade UC. Interestingly, the tumor caused non-islet cell hypoglycemia that was recapitulated in the UC PDX mice [37]. There was no apparent therapeutic effect of treatment with a neutralizing IGF II antibody in the UC PDX model suggesting that the IGF-II production was a paraneoplastic phenomenon and not growth promoting [38].

\section{Application of UC PDX models}

There are a variety of potential applications for the use of PDX in bladder cancer research. For example, PDX have been studied as a tool for precision medicine. While PDX models have been proposed as avatars to predict or personalize therapeutic response in other tumor types, careful studies (i.e. co-clinical trials) have not yet been carried out in UC PDX to support this approach. Perhaps the closest to demonstrating the promise of UC PDX for precision oncology was Pan et al. who showed that their original 22 lines of PDX model morphologically and genetically preserved the trait of the original specimen and performed molecularly targeted therapy with small molecule inhibitors targeting kinases such as HER2 or FGFR3 [14]. Nonetheless, whether the response pattern seen would be reproduced in the patient counterparts is unknown.

Another promising application is to analyze the established PDX models to better understand bladder cancer biology and identify new drug targets. For example, Skowron et al. found that the PDX engraftment allowed amplification of rare basal cell populations with the expression of cell surface markers (CD90+/CD44+/CD49f+), which have tumor-initiating capacity and correlate with prognosis, at similar frequencies when compared to the original donor tumor, while maintaining a heterogenous tumor cell population. They analyzed transcriptome of the sorted basal cells and other differentiated cells which were expanded in the PDX through the process from establishment to serial passages and found that $\mathrm{CDC} 25 \mathrm{C}$ was a prognostic marker in bladder cancer patients [15]. Lee et al. focused on collagen I, which is highly expressed in invasive bladder cancer, and examined a rare bladder cancer PDX model that spontaneously developed lung metastasis to find that bladder tumor cells expressing the collagen receptor, CD167a, responded to collagen I stimulation at the primary tumor to promote local invasion and utilized the same receptor to preferentially colonize at airway smooth muscle 
cells [39]. This result was confirmed in T24 tail vein injection models.

\section{Strategies using complementarity between PDX and $3 D$ culture}

Due to the recent development of 3D culture techniques including organoids and spheroids for bladder cancer, there are a growing number of papers combining 3D culture models and PDX models for complementary purposes. Lee et al. were the first to report a comprehensive panel of UC organoid lines in 2018. They succeeded in establishing organoid lines from transurethral resection (TUR) specimens with a high probability (70\%) as well as conventional PDX tumors, which recapitulated the histopathological and molecular diversity of human bladder cancer and often retained parental tumor heterogeneity [11]. These organoids could be orthotopically transplanted into NSG mice and the in vivo model recapitulated the drug responses observed in organoid culture. Interestingly, it was also noted that a subset of mutations was either lost or gained, or that molecular subtypes were changed, during serial passaging in culture and/or during grafting or reestablishment of organoids from grafts. This finding implies that clonal selection and cell plasticity may depend on the culture environment and promises that organoids will be a tool to accelerate the elucidation of bladder cancer biology.

Spheroid culture is also useful for drug screening and drug target discovery, although it is difficult for long-term subculture. We have recently demonstrated that drug susceptibilities in PDX models were paralleled with corresponding cancer tissue originated spheroid (CTOS) models, established using a 3D culture method by Yoshida et al. [40]. Using multiple clinical specimens, we successfully identified cases in which a combination of disulfiram, an FDA approved drug for alcoholism, and cisplatin was predicted to produce synergy [41]. In another example of the potential superiority of 3D culture, Namekawa et al. showed that ALDH1A1 expression was increased in T24 cells cultured in 3D compared to 2D cultures and that ALDH1 A1 inhibition in patient-derived spheroids and mouse models suppressed the tumor growth [42].

In addition to 3D culture, another recent study described a technology termed conditional reprogramming, which allows efficient establishment of patient tissue-derived 2D cancer cell cultures in the presence of RHO kinase inhibitor and a fibroblast feeder layer [43]. Mondal et al. showed that these conditionally reprogrammed cancer cells established from early passage PDX tumors retain the same STR, genetic alterations and drug sensitivity/resistance profiles. Their proposed strategy for using UC PDX models in combination with the ex vivo culture system should facilitate biobanking and reimplantation as well as high-throughput drug screening and genetic manipulation, which are difficult with UC PDX models alone [44].

\section{Development of humanized mice}

As cancer immunotherapy has come to the center of cancer therapy, PDX models using conventional immunodeficient mice have a serious limitation that the local immune response is not reproduced. However, Wang et al. recently reported that programmed cell death protein 1 (PD-1) inhibitors could be evaluated with hematologically humanized mice [9]. Humanized NSG (HuNSG) mice were generated by transplanting human (h)CD34 $4^{+}$hematopoietic progenitor and stem cells (HSPC) into irradiated NSG mice. The engraftment levels of hCD45+ cells were determined 12 weeks post-HPSC transplantation by flow cytometric quantification of peripheral blood hCD45+ cells. HuNSG mice that had over 25\% hCD45+ cells in the peripheral blood were considered as engrafted and humanized. Treatment with pembrolizumab, which targets PD-1, showed significant growth inhibition in non-small cell lung cancer PDX tumor in HuNSG but not in hematologically unmanipulated NSG mice. Bladder cancer PDX have also been shown to grow in HuNSG mice with partially HLA-matched allogenic human immune systems and showed tumor responses when treated with pembrolizumab. However, in mice that were transplanted HSPC from different donors, pembrolizumab was no longer effective. It is not yet clear what caused it, and further investigation is needed. Blinoba et al. also created NOG / SCID mice transplanted with UC PDX and human lymphocytes from healthy donors at the same time and verified the effect of anti-PD-L1 antibody [10].

\section{DISCUSSION}

The first report on PDX models of urothelial carcinoma was published in 1979 [30]. However, likely due to its low establishment rate and high maintenance costs, this original UC PDX line did not become widely adopted. In the interim, cancer cell lines and CDX models have been widely used to 
study cancer biology and for drug development. Sadly, many candidate drugs while demonstrating promising effects in CDX models do not obtain FDA approval after clinical trials [45-47] highlighting their inadequacy for this purpose. The failures of drug development in phase II and III trials have to some extent been attributed to the low similarity of CDX models to human tumors. For example, cells in CDX tumors are a relatively homogeneous population selected through repeated in vitro passage. Reflecting the need for better pre-clinical models, in combination with the advent of novel immunodeficient mice that improved the engraftment rate, UC PDX are now a more sustainable model that retains intratumoral and interpatient heterogeneity [48-50]. As summarized above, in general UC PDX maintain the genetic background of their donor patient tumors and can also retain patient stromal cells at early passages. In addition, a recent report in colorectal cancer PDX models showed that after the early replacement of the human stroma by murine cells, the murine stroma adopts a human-like metabolic phenotype suggesting that the PDX stroma recapitulates primary tumor stroma in important ways [51]. If a similar adaptation take place in UC PDX, it will also be a great advantage for future translational studies aiming at assessing therapies and concepts that involve both cancer cells and stroma. Given the many papers discussed in this review, PDX will undoubtedly be a very powerful tool for cancer biology studies and drug development in the next decade of UC research.

Among various utilities, use of PDX as a tool for treatment response prediction (i.e. an avatar of the patient) seems to be a promising strategy that is directly relevant to the bedside. Izumchenko et al. reported that an 87\% (112/129) association between therapeutic outcome to chemotherapy and targeted therapy treatment in patients and their corresponding PDX avatar. While this study was across a panel of solid tumors, unfortunately it did not include UC in the response analysis [7]. Their analysis of the PDX drug screens revealed high positive and negative predictive values (PPVs and NPVs) of $85 \%$ and $91 \%$, respectively. Notably, the percentage of correlative therapeutic responses did not vary significantly among different cancer types. A study using UC patients and PDX validating this finding is a first important step in demonstrating that UC PDX are clinically relevant models.

There are significant major barriers to using PDX as avatars for treatment response including but not limited to the variable engraftment rates as well as the cost of generating and testing multiple therapies. Perhaps a complementary model system that bypasses these limitations are the use of 3D culture models exclusively or in combination with PDX models. 3D culture methods are developing rapidly with the aim of constructing in vitro culture systems that better reflect tumor characteristics [52]. The first organoid culture protocol from patientderived tumor tissue was published in the area of colorectal cancer [53, 54]. Since then, organoid culture protocols have been established for many cancer types, and a landmark paper for making bladder cancer organoids were published in 2018 [11]. Like UC PDX, bladder cancer organoids recapitulated the histopathological and molecular diversity of human bladder cancer and often retained parental tumor heterogeneity. These organoids when orthotopically transplanted into NSG mice appear to recapitulate the drug responses observed in organoid culture. Another $3 \mathrm{D}$ culture method, cancer tissue-originated spheroid (CTOS), is a more short term method of 3D culture [55], although it cannot be serially passaged like organoids. The drug susceptibility in the CTOS model also have been shown to be reproduced in the PDX model [41].

These 3D models may complement PDX in drug screening. These models have a short time to establishment, and organoid model can be expanded relatively fast. The reagents required for 3D culture are not cheap, but they are less expensive than establishing, expanding and maintaining PDX. These models also can be cryopreserved, allowing for the generation of living tumor biobanks. Additionally, organoids can be genetically modified [52].

However, 3D culture models also have several limitations. First, since organoids are self-organized structures from dissociated stem cells, it has been reported that there were marked difference in responses to anticancer drugs between organoids even from the same tumor [56]. The fact that organoids are also formed from normal urothelium [57]), and that specific populations can sometimes be selected during establishment and passage [11], suggests the need to thoroughly characterize established organoids and which cell populations are actually expanded. Second, like PDX these 3D culture models do not reproduce the tumor immune microenvironment and in particular lack significant tumor stroma. Therefore, 3D culture models are inadequate to answer biologic questions that revolve around these tumor components and would need to be complemented with corresponding in vivo models 
such as PDX models. We envision that high throughput and low-cost screening can be performed using 3D culture models and then validation and further investigation in vivo using PDX models should follow in translational researches or drug development studies. Together, these preclinical models can reflect the response to anti-cancer therapies and give indications for patient-tailored treatment (Fig. 2). Although the most ideal is to determine the optimal drug based on personalized organoids and PDX, it may be

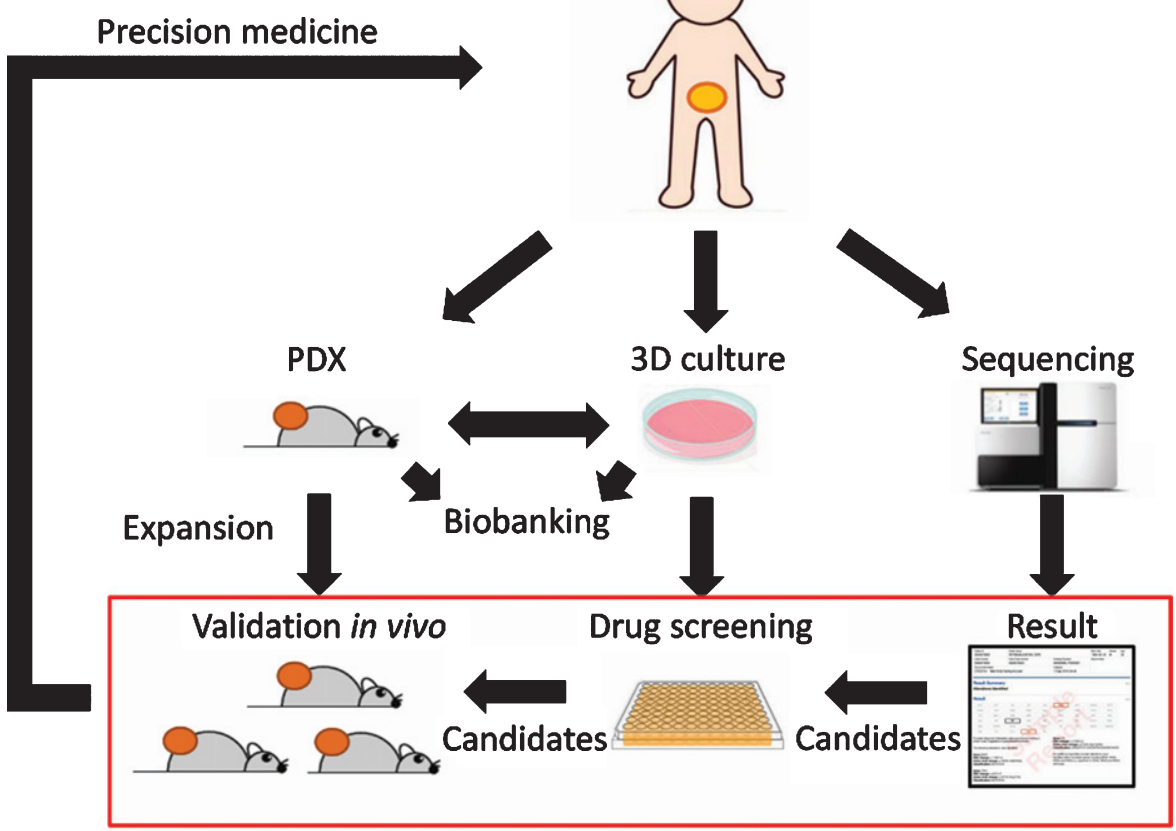

Integrated database

Fig. 2. Schematic representation of combined operation of PDX and 3D culture for precision medicine.

Table 2

Comparison of models using patient specimens

\begin{tabular}{|c|c|c|c|c|c|c|c|c|}
\hline Models & $\begin{array}{l}\text { Establish } \\
\text { rate }\end{array}$ & $\begin{array}{l}\text { Time to } \\
\text { establish }\end{array}$ & $\begin{array}{l}\text { Technical } \\
\text { complexity }\end{array}$ & Scalability & Cost & $\begin{array}{c}\text { Tumor-stoma } \\
\text { interaction }\end{array}$ & $\begin{array}{l}\text { Integrable } \\
\text { immune } \\
\text { system }\end{array}$ & Application \\
\hline $\begin{array}{l}\text { conventional } \\
\text { PDX }\end{array}$ & $-60 \%$ & +++ & + & ++ & ++ & ++ & + & $\begin{array}{l}\text { Validation of candidate drugs } \\
\text { in vivo/ Investigation of } \\
\text { intratumoral heterogeneity/ } \\
\text { Investigation of metastasis } \\
\text { mechanism/ Evaluation of } \\
\text { adoptive T cell therapy in vivo }\end{array}$ \\
\hline $\begin{array}{l}\text { 3D culture } \\
\text { models }\end{array}$ & $70-90 \%$ & + & ++ & +++ & + & + & + & $\begin{array}{l}\text { High throughtput drug } \\
\text { screening for precision cancer } \\
\text { medicine/ Biobanking } \\
\text { Investigation of cancer } \\
\text { stemness and plascity }\end{array}$ \\
\hline $\begin{array}{l}\text { Humanized } \\
\text { mouse } \\
\text { model }\end{array}$ & N.A. & +++ & +++ & + & +++ & +++ & +++ & $\begin{array}{l}\text { Investigation of the } \\
\text { interaction of tumor and } \\
\text { tumor-infiltrating immune } \\
\text { cells/TRANSLATIONAL } \\
\text { studt of immunotherapy } \\
\text { before proceeding to clinical } \\
\text { trials }\end{array}$ \\
\hline
\end{tabular}


difficult to apply progressing UC patients in a clinically relevant time frame. Larger-scale integration of genomic data, functional drug profiling, clinical characteristics, and patient follow-up information into a mineable database also has the potential to inform clinical decisions when personalized tumor cultures are not available by relating genomics to therapeutic responses [58].

The success of immune checkpoint inhibitors in recent years has attracted more attention to immuneoncology. Now, conventional PDX models using immunodeficient mice can be only used as a tool in studies of adoptive $\mathrm{T}$ cell therapy such as chimeric antigen receptor (CAR) - T cell therapy or engineered $\mathrm{T}$ cell therapy, although its therapeutic effect has not yet been shown in urothelial cancer $[19,59]$. The ideal model for investigating mechanisms and predictive biomarkers of response to immune checkpoint inhibition has yet to be defined. But it is clear that PDX and 3D culture models are inadequate. While human immune cells exist at the first engraftment of PDX, they are post-mitotic and are rapidly cleared with serial passages [19]. As for 3D culture models, attempts to overcome this problem by coculture with immune cells have been reported [60], but even this approach does not fully recapitulate the immune response in vivo since these cells primarily represent intratumoral immune cells and ignore the important role of things like antigen priming and memory cell expansion in tumor draining lymph nodes.

In conclusion, PDX platforms have been a major advance in the field of cancer biology and drug development. They importantly appear to retain the histology, genomic characteristics and the drug responsiveness of the corresponding patient tumors making them faithful patient avatars. Were it not for the recent recognition of the importance of anti-tumor immunity in cancer therapy, the relevance and use of PDX models might have continued their expansion. The coming years will likely sort out the utility of PDX models generated in the context of mice with a humanized immune system for studying the immune microenvironment and this result will likely determine whether there is an increase or decrease in demand for these models.

\section{ACKNOWLEDGMENTS}

The authors have no acknowledgments.

\section{FUNDING}

This study was partly supported by Grant-in-aid from the Ministry of Education and Science (to T.K. \#25713055 and \#19H03790, to O.O. \#26253078), the University Cancer Research Fund (UCRF) (to W.Y.K.), the American Cancer Society Grant (to W.Y.K. RSG-14-219-01-TBG) and Thomas M. Mohr Fund for Bladder Cancer Research (to W.Y.K.).

\section{AUTHOR CONTRIBUTIONS}

Concept: YK, OO and T Kobayashi; Search: YK; Writing: YK, RS, TI, TK and WYK.

\section{ETHICAL CONSIDERATIONS}

This study, as a literature review is exempt from any requirement for Institutional Review Board approval.

\section{CONFLICT OF INTEREST}

WYK: Stock and Other Ownership Interests Abbvie, Abbott, Amgen, BeiGene, Bristol-Myers Squibb, FibroGen, Illumina, Johnson \& Johnson, NextCure, Oramed; Honoraria - H3 Biomedicine, Takeda; Consulting or Advisory Role - Foundation Medicine, GeneCentric; Research Funding - Acerta, Foundation Medicine, GeneCentric, Merck; Patents, Royalties, Other Intellectual Property - Inventor: BASE47 Subtype Classifier; Travel, Accommodations, Expenses - H3 Biomedicine, Takeda.

The other authors have no conflict of interest to declare.

\section{REFERENCES}

[1] Bray F, Ferlay J, Soerjomataram I, Siegel RL, Torre LA, Jemal A. Global cancer statistics 2018: GLOBOCAN estimates of incidence and mortality worldwide for 36 cancers in 185 countries. CA: a Cancer Journal for Clinicians. 2018;68(6):394-424.

[2] Stenzl A, Cowan NC, De Santis M, Kuczyk MA, Merseburger AS, Ribal MJ, et al. Treatment of muscle-invasive and metastatic bladder cancer: update of the EAU guidelines. Eur Urol. 2011;59(6):1009-18.

[3] Bellmunt J, de Wit R, Vaughn DJ, Fradet Y, Lee JL, Fong L, et al. Pembrolizumab as Second-Line Therapy for Advanced Urothelial Carcinoma. N Engl J Med. 2017;376(11):101526.

[4] Hidalgo M, Amant F, Biankin AV, Budinska E, Byrne AT, Caldas C, et al. Patient-derived xenograft models: an emerging platform for translational cancer research. Cancer Discov. 2014;4(9):998-1013. 
[5] Tentler JJ, Tan AC, Weekes CD, Jimeno A, Leong S, Pitts TM, et al. Patient-derived tumour xenografts as models for oncology drug development. Nat Rev Clin Oncol. 2012;9(6):338-50.

[6] Inoue T, Terada N, Kobayashi T, Ogawa O. Patientderived xenografts as in vivo models for research in urological malignancies. Nat Rev Urol. 2017;14(5): 267-83.

[7] Izumchenko E, Paz K, Ciznadija D, Sloma I, Katz A, Vasquez-Dunddel D, et al. Patient-derived xenografts effectively capture responses to oncology therapy in a heterogeneous cohort of patients with solid tumors. Ann Oncol. 2017;28(10):2595-605.

[8] Gao H, Korn JM, Ferretti S, Monahan JE, Wang Y, Singh $\mathrm{M}$, et al. High-throughput screening using patient-derived tumor xenografts to predict clinical trial drug response. Nat Med. 2015;21(11):1318-25.

[9] Wang M, Yao LC, Cheng M, Cai D, Martinek J, Pan CX, et al. Humanized mice in studying efficacy and mechanisms of PD-1-targeted cancer immunotherapy. FASEB J. 2018;32(3):1537-49.

[10] Blinova E, Roshchin D, Kogan E, Samishina E, Demura T, Deryabina O, et al. Patient-Derived Non-Muscular Invasive Bladder Cancer Xenografts of Main Molecular Subtypes of the Tumor for Anti-Pd-11 Treatment Assessment. Cells. 2019;8(6).

[11] Lee SH, Hu W, Matulay JT, Silva MV, Owczarek TB, Kim K, et al. Tumor Evolution and Drug Response in Patient-Derived Organoid Models of Bladder Cancer. Cell. 2018;173(2):515-28.e17.

[12] Liberati A, Altman DG, Tetzlaff J, Mulrow C, Gotzsche PC, Ioannidis JP, et al. The PRISMA statement for reporting systematic reviews and meta-analyses of studies that evaluate health care interventions: explanation and elaboration. PLoS Medicine. 2009;6(7):e1000100.

[13] Abe T, Tada M, Shinohara N, Okada F, Itoh T, Hamada J, et al. Establishment and characterization of human urothelial cancer xenografts in severe combined immunodeficient mice. Int J Urol. 2006;13(1):47-57.

[14] Pan CX, Zhang H, Tepper CG, Lin TY, Davis RR, Keck J, et al. Development and Characterization of Bladder Cancer Patient-Derived Xenografts for Molecularly Guided Targeted Therapy. PLoS One. 2015;10(8): e0134346.

[15] Skowron KB, Pitroda SP, Namm JP, Balogun O, Beckett MA, Zenner ML, et al. Basal Tumor Cell Isolation and Patient-Derived Xenograft Engraftment Identify High-Risk Clinical Bladder Cancers. Sci Rep. 2016;6:35854.

[16] Alkema NG, Tomar T, Duiker EW, Jan Meersma G, Klip $H$, van der Zee $A G$, et al. Biobanking of patient and patient-derived xenograft ovarian tumour tissue: efficient preservation with low and high fetal calf serum based methods. Sci Rep. 2015;5:14495.

[17] Porter LH, Lawrence MG, Wang H, Clark AK, Bakshi A, Obinata D, et al. Establishing a cryopreservation protocol for patient-derived xenografts of prostate cancer. Prostate. 2019;79(11):1326-37.

[18] Ivanics T, Bergquist JR, Liu G, Kim MP, Kang Y, Katz MH, et al. Patient-derived xenograft cryopreservation and reanimation outcomes are dependent on cryoprotectant type. Lab Invest. 2018;98(7):947-56.

[19] Saito R, Kobayashi T, Kashima S, Matsumoto K, Ogawa O. Faithful preclinical mouse models for better translation to bedside in the field of immuno-oncology. Int J Clin Oncol. 2019.
[20] Park B, Jeong BC, Choi YL, Kwon GY, Lim JE, Seo SI, et al. Development and characterization of a bladder cancer xenograft model using patient-derived tumor tissue. Cancer Sci. 2013;104(5):631-8.

[21] Jager W, Xue H, Hayashi T, Janssen C, Awrey S, Wyatt AW, et al. Patient-derived bladder cancer xenografts in the preclinical development of novel targeted therapies. Oncotarget. 2015;6(25):21522-32.

[22] Roife D, Kang Y, Wang L, Fang B, Swisher SG, Gershenwald JE, et al. Generation of patient-derived xenografts from fine needle aspirates or core needle biopsy. Surgery. 2017;161(5):1246-54.

[23] Chan KS, Espinosa I, Chao M, Wong D, Ailles L, Diehn M, et al. Identification, molecular characterization, clinical prognosis, and therapeutic targeting of human bladder tumor-initiating cells. Proc Natl Acad Sci U S A. 2009;106(33):14016-21.

[24] Wang W, Iyer NG, Tay HT, Wu Y, Lim TK, Zheng L, et al. Microarray profiling shows distinct differences between primary tumors and commonly used preclinical models in hepatocellular carcinoma. BMC Cancer. 2015;15:828.

[25] Wilmanns C, Fan D, O’Brian CA, Bucana CD, Fidler IJ. Orthotopic and ectopic organ environments differentially influence the sensitivity of murine colon carcinoma cells to doxorubicin and 5-fluorouracil. Int J Cancer. 1992;52(1):98104.

[26] Gills J, Moret R, Zhang X, Nelson J, Maresh G, Hellmers L, et al. A patient-derived orthotopic xenograft model enabling human high-grade urothelial cell carcinoma of the bladder tumor implantation, growth, angiogenesis, and metastasis. Oncotarget. 2018;9(66):32718-29.

[27] Zhao Y, An MJ, Zhang H, Tan DX, Chen X, Wu PP, et al. Patient-derived bladder cancer xenograft models reveal VEGF and CDK4 enhancing tumor metastasis behavior. RSC Adv. 2019;9(31):17877-84.

[28] Kovnat A, Buick RN, Connolly JG, Jewett MA, Keresteci AG, Tannock IF. Comparison of growth of human bladder cancer in tissue culture or as xenografts with clinical and pathological characteristics. Cancer Res. 1984;44(6): 2530-3.

[29] Bondarenko G, Ugolkov A, Rohan S, Kulesza P, Dubrovskyi O, Gursel D, et al. Patient-Derived Tumor Xenografts Are Susceptible to Formation of Human Lymphocytic Tumors. Neoplasia (New York, NY). 2015;17(9):735-41.

[30] Sufrin G, McGarry MP, Sandberg AA, Murphy GP. Heterotransplantation of human transitional cell carcinoma in athymic mice. J Urol. 1979;121(2):159-61.

[31] Russell PJ, Raghavan D, Gregory P, Philips J, Wills EJ, Jelbart M, et al. Bladder cancer xenografts: a model of tumor cell heterogeneity. Cancer Res. 1986;46(4 Pt 2): 2035-40.

[32] Hay JH, Busuttil A, Steel CM, Duncan W. The growth and histological characteristics of a series of human bladder cancer xenografts. Radiother Oncol. 1986;7(4):331-40.

[33] McCue PA, Gomella LG, Veltri RW, Marley GM, Miller MC, Lattime EC. Development of secondary structure, growth characteristics and cytogenetic analysis of human transitional cell carcinoma xenografts in scid/scid mice. J Urol. 1996;155(3):1128-32.

[34] Chang N, Lee HW, Lim JE, Jeong DE, Song HJ, Kim $\mathrm{S}$, et al. Establishment and antitumor effects of dasatinib and PKI-587 in BD-138T, a patient-derived muscle invasive bladder cancer preclinical platform with concomitant EGFR amplification and PTEN deletion. Oncotarget. 2016;7(32):51626-39. 
[35] Wei L, Chintala S, Ciamporcero E, Ramakrishnan S, Elbanna M, Wang $\mathbf{J}$, et al. Genomic profiling is predictive of response to cisplatin treatment but not to PI3K inhibition in bladder cancer patient-derived xenografts. Oncotarget. 2016;7(47):76374-89.

[36] Hofner T, Macher-Goeppinger S, Klein C, Rigo-Watermeier T, Eisen C, Pahernik S, et al. Development and characteristics of preclinical experimental models for the research of rare neuroendocrine bladder cancer. J Urol. 2013;190(6):2263-70.

[37] Samaratunga H, Delahunt B, Egevad L, Adamson M, Hussey D, Malone G, et al. Pleomorphic giant cell carcinoma of the urinary bladder: an extreme form of tumour de-differentiation. Histopathology. 2016;68(4):533-40.

[38] Funada S, Kita Y, Okada Y, Kobayashi T, Teramoto Y, Shibuya $\mathrm{S}$, et al. Bladder urothelial carcinoma producing insulin-like growth factor II: A case report. IJU Case Reports. 2018;1(1):9-12.

[39] Lee YC, Kurtova AV, Xiao J, Nikolos F, Hayashi K, Tramel $\mathrm{Z}$, et al. Collagen-rich airway smooth muscle cells are a metastatic niche for tumor colonization in the lung. Nat Commun. 2019;10(1):2131.

[40] Yoshida T, Okuyama H, Endo H, Inoue M. Spheroid Cultures of Primary Urothelial Cancer Cells: Cancer Tissue-Originated Spheroid (CTOS) Method. Methods in molecular biology (Clifton, NJ). 2018;1655:145-53.

[41] Kita Y, Hamada A, Saito R, Teramoto Y, Tanaka R, Takano $\mathrm{K}$, et al. Systematic chemical screening identifies disulfiram as a repurposed drug that enhances sensitivity to cisplatin in bladder cancer: a summary of preclinical studies. Br J Cancer. 2019;121(12):1027-1038.

[42] Namekawa T, Ikeda K, Horie-Inoue K, Suzuki T, Okamoto $\mathrm{K}$, Ichikawa T, et al. ALDH1A1 in patient-derived bladder cancer spheroids activates retinoic acid signaling leading to TUBB 3 overexpression and tumor progression. Int J Cancer. 2020;15;146(4):1099-1113.

[43] Liu X, Ory V, Chapman S, Yuan H, Albanese C, Kallakury $\mathrm{B}$, et al. ROCK inhibitor and feeder cells induce the conditional reprogramming of epithelial cells. Am J Pathol. 2012;180(2):599-607.

[44] Mondal AM, Ma AH, Li G, Krawczyk E, Yuan R, Lu J, et al. Fidelity of a PDX-CR model for bladder cancer. Biochem Biophys Res Commun. 2019;517(1):49-56.

[45] Johnson JI, Decker S, Zaharevitz D, Rubinstein LV, Venditti JM, Schepartz S, et al. Relationships between drug activity in NCI preclinical in vitro and in vivo models and early clinical trials. Br J Cancer. 2001;84(10): 1424-31.

[46] Sharpless NE, Depinho RA. The mighty mouse: genetically engineered mouse models in cancer drug development. Nat Rev Drug Discov. 2006;5(9):741-54.

[47] Hwang TJ, Carpenter D, Lauffenburger JC, Wang B, Franklin JM, Kesselheim AS. Failure of Investiga- tional Drugs in Late-Stage Clinical Development and Publication of Trial Results. JAMA Internal Medicine. 2016;176(12):1826-33.

[48] Eirew P, Steif A, Khattra J, Ha G, Yap D, Farahani $\mathrm{H}$, et al. Dynamics of genomic clones in breast cancer patient xenografts at single-cell resolution. Nature. 2015;518(7539):422-6.

[49] Nguyen LV, Cox CL, Eirew P, Knapp DJ, Pellacani D, Kannan N, et al. DNA barcoding reveals diverse growth kinetics of human breast tumour subclones in serially passaged xenografts. Nat Commun. 2014;5:5871.

[50] Cassidy JW, Caldas C, Bruna A. Maintaining Tumor Heterogeneity in Patient-Derived Tumor Xenografts. Cancer Res. 2015;75(15):2963-8.

[51] Blomme A, Van Simaeys G, Doumont G, Costanza B, Bellier J, Otaka Y, et al. Murine stroma adopts a human-like metabolic phenotype in the PDX model of colorectal cancer and liver metastases. Oncogene. 2018;37(9):1237-50.

[52] Bleijs M, van de Wetering M, Clevers H, Drost J. Xenograft and organoid model systems in cancer research. EMBO J. 2019;38(15):e101654.

[53] Sato T, Vries RG, Snippert HJ, van de Wetering M, Barker N, Stange DE, et al. Single Lgr5 stem cells build crypt-villus structures in vitro without a mesenchymal niche. Nature. 2009;459(7244):262-5.

[54] Sato T, Stange DE, Ferrante M, Vries RG, Van Es $\mathrm{JH}$, Van den Brink $\mathrm{S}$, et al. Long-term expansion of epithelial organoids from human colon, adenoma, adenocarcinoma, and Barrett's epithelium. Gastroenterology. 2011;141(5):1762-72.

[55] Okuyama H, Yoshida T, Endo H, Nakayama M, Nonomura N, Nishimura K, et al. Involvement of heregulin/HER3 in the primary culture of human urothelial cancer. J Urol. 2013;190(1):302-10.

[56] Roerink SF, Sasaki N, Lee-Six H, Young MD, Alexandrov LB, Behjati $\mathrm{S}$, et al. Intra-tumour diversification in colorectal cancer at the single-cell level. Nature. 2018;556(7702):457-62.

[57] Mullenders J, de Jongh E, Brousali A, Roosen M, Blom JPA, Begthel H, et al. Mouse and human urothelial cancer organoids: A tool for bladder cancer research. Proc Natl Acad Sci U S A. 2019;116(10):4567-74.

[58] Pauli C, Hopkins BD, Prandi D, Shaw R, Fedrizzi T, Sboner A, et al. Personalized In Vitro and In Vivo Cancer Models to Guide Precision Medicine. Cancer Discov. 2017;7(5): 462-77.

[59] Deng C, Zhao J, Zhou S, Dong J, Cao J, Gao J, et al. The Vascular Disrupting Agent CA4P Improves the Antitumor Efficacy of CAR-T Cells in Preclinical Models of Solid Human Tumors. Mol Ther. 2020;28(1):75-88.

[60] Neal JT, Li X, Zhu J, Giangarra V, Grzeskowiak CL, Ju J, et al. Organoid Modeling of the Tumor Immune Microenvironment. Cell. 2018;175(7):1972-88.e16. 